SALUD

Ciencias sociales

HUMANIDADES

MAS ALLÁ DE LOS COMITÉS DE ÉTICAASISTENCIAL:

HACIA UNA CULTURA BIOÉTICA

-BEGOÑA ROMÁN 1

\title{
PENSAMIENTO ACTUAL
}

PROLEGÓMENOS PARA UNA FILOSOFÍA DE LA ENFERMEDAD.

- MARCO SANZ.

JOSÉ DE LETAMENDI, FUNDAMENTO DE SU CIENCIA Y CAUSA DE SU DESPRESTIGIO

- EMILIO CERVANTES 36

\section{ARTE, SALUD Y SOCIEDAD}

TODOS POSEEMOS INTELIGENCIA MUSICAL 
Co-directores

Marc Antoni Broggi i Trias (PCBC)

Francesc Borrell (UB)

\section{Jefa de Redacción}

Núria Estrach (UAB)

\section{Consejo científico}

Juan Carlos Hernández Clemente

Juan Medrano Albéniz

Vicente Morales Hidalgo

\section{Correspondencia \\ Web:}

http://www.fundacionletamendi.com

Correo electrónico:

info@fundacionletamendi.com

\section{Envío de manuscritos:}

http://www.fundacionletamendi.com/revista-foliahumanistica/envio-de-manuscritos/

\section{Información editorial}

Folia Humanística publica artículos por encargo solicitados a especialistas, así como aquellas propuestas enviadas por los autores y aceptadas tras su evaluación por pares de académicos especializados.

Los textos recibidos se publicarán en la lengua original (castellano, catalán, inglés y francés); los que se consideren de relevancia mayor serán traducidos al inglés y castellano.

Los artículos deben ser originales y acompañados del documento "derechos de autor" que encontrarán en la web, junto a las normas de presentación a seguir.

Cada artículo publicado al final tendrá especificado la referencia de citación, donde se incluirá el número $\mathrm{DOI}{ }^{\circledR}$.

\section{Distribución}

La Revista Folia Humanística es de libre acceso a consultar online.

http://www.fundacionletamendi.com/category/rev

istal

Folia Humanística es una revista internacional que tiene el doble objetivo de fomentar, por un lado, la reflexión y el debate público en el ámbito de la Salud, Ciencias Sociales y Humanidades, y por el otro, la colaboración entre distintos equipos de investigación nacionales e internacionales que dinamicen el diálogo entre la filosofía de la medicina, la salud pública y la justicia social. Dividida en "Tema del día", (artículos para el debate), "Pensamiento actual", (artículos críticos de novedades editoriales), y "Arte, Salud y Sociedad", la revista se esfuerza en fortalecer las conexiones entre la investigación académica, la práctica clínica, las experiencias de los pacientes y sus implicaciones éticas y estéticas en la sociedad. Todo ello con la intención de favorecer la reflexión entre diferentes disciplinas sobre temas de actualidad y las tendencias más novedosas en el campo de las Humanidades y la Salud.

Folia Humanística is an International Journal, born with the dual aim of fuelling the discussion and public debate on issues of health, social sciences and humanities and on the hand, of fostering cooperation between various research groups, both national and International, to spur the dialogue between philosophy and medicine, public health and social justice. The Journal is divided into three different sections: "main focus" (article for debate), "Contemporary thought" (critical reviews of new Publications) and "Arts, Health and Society" which all contribute to strengthening the links between academic research, clinical practice, the experience of patients and their ethical and esthetical implications for society. Ultimately, the intention of the Journal is to promote reflection at the crossroads of several disciplines on topical issues and new trends in humanities and health. 


\title{
MÁS ALLÁ DE LOS COMITÉS DE ÉTICA ASISTENCIAL: HACIA UNA CULTURA BIOÉTICA
}

\section{Begoña Román Maestre}

\begin{abstract}
Resumen: Más allá de los Comités de ética asistenciales (CEAs), que no necesariamente padecen el síndrome de retraso en el crecimiento, aquí exponemos una visión crítica del quehacer de la bioética clínica en aras de mejorar su desarrollo. Partimos de la base de que lo que falla no son los CEA's, ni los profesionales que se dedican a la bioética, sino la ausencia de cultura. $Y$ esta ausencia puede hacer fracasar muchas iniciativas (el modelo centrado en la persona, el de decisiones compartidas, etc.) debido a la falta de arraigo en una cultura bioética.

Si bien se ha hecho mucho en y por la formación en bioética entre los profesionales, ésta se resiente en la implementación de los cambios que recomienda. En bioética no hemos logrado dialogar más allá de los comités, no hemos impactado substancialmente en la organización, ni tampoco en la ciudadanía. Existe un gran riesgo de que la bioética devenga una especie de hongo en medio de un terreno indiferente e incluso baldío. Proponemos ampliar la mirada de la bioética clínica hacia la forja de una cultura, ineludible para su saludable desarrollo. La bioética también precisa poder, autoridad y liderazgo. El coraje para llevar a cabo los cambios bioéticos, o para asumir que no se puede sin otros apoyos, son virtudes que no solo son exigibles a los profesionales, sino también a organizaciones, gestores y políticos.
\end{abstract}

En concreto, abordaremos tres dificultades que esta carencia cultural genera: 1) La complejidad propia del diálogo multi e interdisciplinar (nivel profesional). 2) La flexibilidad y coordinación, necesarias para atender al contexto, precisan de un determinado tipo de organizaciones e instituciones (nivel organizativo). 3) La derivada de la participación de la ciudadanía (nivel cívico).

Palabras clave: bioética /cultura/ comités de ética.

\section{Abstract: PATIENT SAFETY IN HOSPITAL CARE: ARE WE ADDRESSING IT FROM THE RIGHT PERSPECTIVE?}

Beyond Hospital Ethics Committees (HECs), that they do not necessary suffer from Failure to Thrive syndrome, here we expose a critical vision of the work of clinical bioethics in order to improve its development. We start from the basis that what fails is not the HECs, nor the professionals dedicated to bioethics, but the absence of culture. And this absence can make failure may initiatives (centered-patient model, shared decisions model, etc.) due to lack of roots in a bioethical culture.

Although much has been done in and for the training in bioethics among professionals, it resents the implementation of the changes it recommends. In bioethics we have not managed to dialogue beyond the committees, we have not substantially impacted on the organization, nor on citizenship. There is a great risk that bioethics will become a kind of fungus in the middle of an indifferent context and even wasteland. We propose to broaden the look of clinical bioethics to the forging of a culture, which is unavoidable for its healthy development. Bioethics also needs power, authority and leadership. The courage to carry out bioethical changes, or to assume that it cannot be done without other support, are virtues that are not only required to professionals, but also of organizations, managers and politicians.

Specifically, we will address three difficulties that this cultural lack generates:1) The complexity of multi and interdisciplinary dialogue (professional level). 2) The flexibility necessary to meet the precise context of a certain type of organizations and institutions (organizational level). 3) The derivative of citizen participation (civic level). 
Keywords: bioethics/ Culture/ Committees.

Artículo recibido: 2 mayo 2020; aceptado: 1 junio 2020.

\section{NECESIDAD DE UNA CULTURA BIOÉTICA}

Con ánimo de hacer balance y rendimiento de cuentas, al observar el desarrollo de la bioética en España, podemos constatar unos haberes y unos deberes.

Entre los haberes, y viniendo de la nada, podemos congratularnos de lo mucho que se ha hecho: formación de profesionales a todos los niveles, de grado y postgrado (sobre todo de enfermería y medicina); la forja y desempeño de los comités en sus distintas variables (asistenciales, de investigación, autonómicos y nacionales). Gran parte de este éxito se lo debemos al esfuerzo de personas entusiastas y muy preparadas que fueron precursores y ejercieron un gran liderazgo (Francesc Abel, Diego Gracia, Javier Gafo, Victoria Camps, Adela Cortina, María Casado) ${ }^{1}$. Conscientes de que la bioética exigía un cambio cultural, comenzaron por lo esencial: (trans)formar las mentalidades de profesionales y organizaciones requería formación continua. Por ello crearon institutos, fundaciones, y promovieron los primeros cursos y másteres en bioética. Se impartió formación, se escribió mucho y de calidad, se institucionalizaron los comités ${ }^{2}$ y se llevaron a cabo importantes cambios legislativos con clara impronta bioética ${ }^{3}$.

Comenzábamos de cero y había que crear e instituir: ser actores y autores. Pero, como nos advirtiera Arendt, es el narrador el que hace historia (Arendt, 2016),

\footnotetext{
${ }^{1}$ Ellos están detrás de instituciones hoy tan reconocidas en la Bioética española como son el Instituto Borja de Bioética, la Fundación Ciencias de la Salud, la Universidad de Comillas, la Fundación Víctor Grífols i Lucas, o el Observatorio de Bioética y Derecho de la UB, entre otras.

2 Recordemos el Simposio de abril de 1991 en Barcelona "La Bioética i els seus Comitès", entre cuyas conclusions mencionaremos: 1-necesidad de formar a profesionales en el campo de la bioètica; 2necesidad de acreditar comitès hospitalarios y 3-necesidad de crear una instancia central pluridisciplinària,(entidad que se plasmaria finalment en el Comitè de Bioètica de Catalunya); (Ver: Marc-Antoni Broggi i Francesc Abel. La bioètica a Catalunya. Salut Catalunya, 1991; 5:149-51).

${ }^{3}$ Las leyes "de Autonomía del Paciente" tuvieron su origen en el debate bioético, (año 2001 en Cataluña y en el resto de España después, siguiendo la estela del "Convenio" del CAHBI del Consejo de Europa).
} 


FOLIA HUMANÍSTICA, Revista de Salud, ciencias sociales y humanidades
Núm.2 (Vol. 2) junio-julio 2020. ISSN 2462-2753

porque es el que tiene la suficiente perspectiva de lo recorrido, sabiendo de dónde viene, lo que se ha hecho más o menos acertadamente, para vislumbrar hacia dónde quiere ir en busca de un horizonte de sentido hacia el cual continuar y por el cual perseverar. En esa línea, si aquí y ahora asumimos el rol de narradores de lo hecho, no lo hacemos, en absoluto, ni con ánimo exhaustivo ni de juzgar, sino con el deseo de orientarnos y, desde el deber de gratitud, encontrar el sentido. La mera autocomplacencia no ayuda a crecer.

A pesar de que se ha hecho un gran trabajo, y que podemos presumir de tener comités y de la calidad de sus recomendaciones, sin embargo, el impacto de éstas, o las consultas que llegan a los comités, son escasas. No ha sido fluida la relación con los Departamentos de Salud y los Parlamentos; como tampoco ha sido buena la coordinación con otros servicios u otros Departamentos (servicios sociales, educación, justicia, etc.). Por eso nos hallamos de nuevo discutiendo temas que pensábamos que teníamos resueltos (consentimiento informado, vacunas, eutanasia, etc.). Nos sentimos como estancados en el día de la marmota, igual que el personaje de la película "Atrapado en el tiempo". Todo ello provoca, haciendo un guiño a Freud, un cierto malestar en la cultura solo que, precisamente esa es nuestra tesis, creemos obedece a la falta de cultura bioética.

Ya sabíamos que la formación en bioética de los profesionales no les generaba más satisfacción, sino todo lo contrario, les condenaba a un stress moral fruto de la mayor consciencia de cómo se deben hacer las cosas frente a cómo se están haciendo. Estudiados están los fenómenos del estrés en los profesionales que ven la incoherencia entre lo que se debe y lo que se hace (Falcó, 2012). Hay cierta sensación entre los que nos hemos dedicado a la bioética ${ }^{4}$ de que no nos han hecho caso del todo, o de que no hemos sabido convencer. Otras veces, en cambio, hemos sucumbido a la tentación de convertir las recomendaciones en leyes, o en protocolos,

\footnotetext{
${ }^{4}$ Estoy en deuda con el seminario que realizamos en Vic, en enero del 2019 en las vísperas del I Congreso internacional de Bioética de la Universidad Central de Cataluña; especialmente sugerente para estas reflexiones me resultó la conferencia de la Dra. Lydia Feyto.
} 


FOLIA HUMANÍSTICA, Revista de Salud, ciencias sociales y humanidades
Núm.2 (Vol. 2) junio-julio 2020. ISSN 2462-2753

o en incentivos en la dirección por objetivos, o en medidas cuantitativas, como si fueran las únicas maneras de lograr ser eficiente.

Sin duda, conseguir que algo recomendado éticamente como bueno sea obligatorio por ley ayuda, pero no siempre; a veces se puede volver en contra del propósito. Pensemos, por ejemplo, en el caso del consentimiento informado. Más que un documento a firmar alude al proceso comunicativo por el que el paciente, en su derecho a ser informado, recibe la pertinente información para poder ejercer su autonomía sobre a qué tratamientos o intervenciones someterse, dados los riesgos y beneficios. El consentimiento se recibió como un triunfo de la bioética en cuanto era un reconocimiento al principio de respeto a la autonomía del paciente, a su derecho a participar, y al deber de los profesionales de informarlo, en aras de lograr unas decisiones lo más compartidas posible por bien acompañadas.

Pero no es ni riguroso ni responsable sin más "hacer trasplantes", trasladando aquí algo que funcionaba (?) en USA. Había que atender al contexto profesional, organizativo, político. Cuando el consentimiento llega a España no se pensó en las posibilidades reales de hacerlo bien, es decir, no se prestó suficiente atención a los cambios organizativos que implicaba: la formación de los profesionales que requería, así como la coordinación entre profesionales y servicios, o la pedagogía a la ciudadanía, dado el nuevo rol de agente de salud responsable de la toma de decisiones. El mero documento del consentimiento informado no podía suplir todo eso.

En un contexto cultural como el español, el consentimiento informado se ha acabado convirtiendo en papel impreso, redactado por abogados y profesionales, con ánimo defensivo, explicitando todos los posibles riesgos; a toda prisa se hace firmar a los pacientes, cual mero trámite. Y todavía discutimos si se necesita la firma de los profesionales y, en su caso, cuál de ellos, si el que pide la prueba o el que la realiza. Todo ello queda muy lejos de la manera dialógica y deliberativa, que es la propia de la bioética. Los mismos profesionales asistenciales no fueron conscientes de lo corrosivo de ese proceso; tampoco fueron capaces de alertar sobre la imposibilidad 
de llevarlo a la práctica en la forma y modo que se les requería desde la cultura bioética. A otros muchos, que sí se pronunciaron, no se les escuchó, o no se les quiso comprender ${ }^{5}$. El caso del consentimiento informado evidencia que el espíritu de la medicina defensiva venció al de la centralidad del paciente, la importancia de granjearse su confianza y promover su participación. Quedó en terreno baldío.

Lo que se hizo en bioética pues era necesario, pero no fue suficiente: no se ha hecho la (trans)formación de la cultura. Cambiar superficialmente para seguir haciendo lo mismo es un simulacro, un sucedáneo que no va a servir ni para paliar el malestar ${ }^{6}$. No se trataba solo de mejorar las conductas y los protocolos, se trata de querer hacer y saber por qué hacerlo. Cambiar las prácticas sin que se haya producido un profundo cambio cultural, puede comportar un mecanicismo en las maneras de hacer y un conservadurismo que no se aviene con el espíritu bioético, que es crítico y reflexivo por definición.

La ética, aparte de una reflexión crítico-racional sobre las costumbres o mores, alude a una manera de ser y estar en el mundo, en nuestro tiempo y con /entre otros. De eso va la ética aplicada: de crear cultura, regenerando morales y creando otras. $Y$ ¿qué hace falta para forjar una cultura? Ineludiblemente, el querer forjarla, discutir cómo la queremos y que cada uno asuma la parte que le corresponde desde su poder y, por tanto, desde su responsabilidad. Entre nuestros deberes bioéticos debería constar ante todo un activismo biopolítico para cambiar prácticas, mentalidades e instituciones. En efecto, la bioética es más que una asignatura, su formación exige de prácticas, instituciones, comunidad, y de personas referentes (Maclntyre, 1987).

\footnotetext{
${ }^{5}$ Agradezco al Dr. Francesc Borrell estas reflexiones sobre el consentimiento informado.

${ }^{6}$ Así también ha acontecido con la responsabilidad social corporativa en el ámbito de la ética empresarial: no bastaba con añadirla sin cambiar profundamente la forma de concebir la empresa.
} 


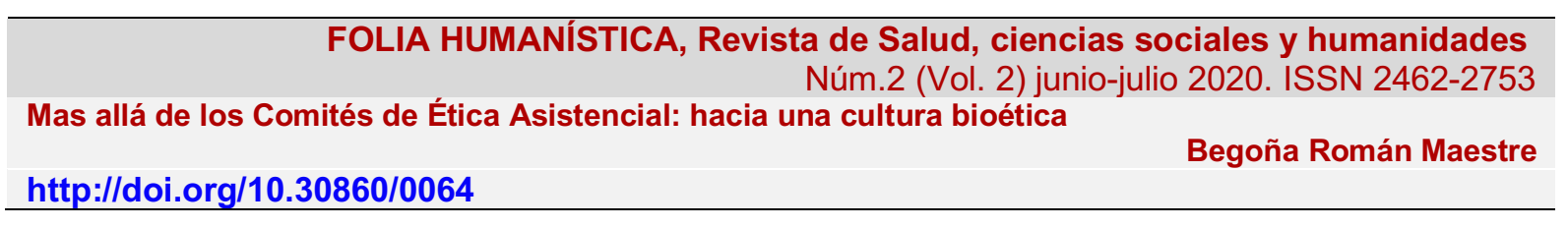

Como la define la Real Academia Española, la cultura es el conjunto de conocimientos que permite a alguien desarrollar su juicio crítico. En una tercera acepción, se dice que la cultura es el conjunto de modos de vida y costumbres, conocimientos y grado de desarrollo artístico, científico, industrial, en una época, grupo social, etc. Aplicando estas definiciones a nuestro ámbito específico de la cultura bioética, precisamos de la formación de los profesionales en la capacidad de duda, de crítica y de pensar alternativas, es decir, diferentemente, para desarrollar ese juicio crítico. Pero luego hay que construir, y esto es difícil en entornos laborales tan asistencialmente cargados, con poco tiempo para pararse a pensar. El equilibrio malabar que hay que lograr para que los servicios funcionen en el día a día deja poco margen de maniobra a la alternativa, a la duda, al juicio crítico. Y el poco que deja sólo sirve para aumentar el malestar de aquellos profesionales que, conscientes de la necesidad de hacerlo mejor, se hallaban solos, impotentes o no reconocidos.

La misma etimología de cultura alude al cultivo, a la necesidad de preparar el terreno (la agricultura sabe de ciclos, ritmos y cuidados). Hemos hecho mucho en formación de profesionales y en la institucionalización de comités de ética, pero tememos que todo ello se quedó un tanto aislado y un tanto ineficiente. Si hay que volver sobre los mismos temas es, a veces, porque no difundimos bien lo que dijimos, pero también y sobre todo porque el cambio cultural no se produjo: se añadió el comité de ética, pero no cuajó en lo trascendental: no se aplicó en serio.

La bioética es demandada en una sociedad con desajustes entre las técnicas, las expectativas y derechos y deberes de la ciudadanía, los responsables políticos y las posibilidades reales, que siempre son limitadas. La bioética versa sobre problemas trágicos, en el sentido de que no hay una solución buena y clara, sino que, como en el caso de los dilemas, en ambas posibilidades se exponen buenas razones. $Y$ además hay muchos actores involucrados. E. O. Wilson nos alertaba del gran problema que es para los humanos tener emociones paleolíticas, instituciones medievales y tecnología de dioses (Wilson, 2011). Los humanos somos animales de costumbres y nuestras instituciones son medievales en su quehacer. Para tamaña 
tarea de crear una cultura bioética hace falta un gran equipo que piensa y coordinación para su aplicación.

La cultura bioética exige creatividad e innovación que no son individuales, sino fruto de tareas colectivas y cooperativas. Pensar juntos pasa por demostrar por qué razones concedemos hoy mayor importancia a unas recomendaciones e intervenciones que a otras. Sin embargo, luego hay que poner manos a la obra. Más allá del caso particular, más o menos anecdótico, hemos de ser capaces de generar categorías culturales, y cultura de categoría. Por eso son cruciales las buenas prácticas, en tanto que implican emprendimiento de nuevas acciones y maneras de hacer, así como dejar de hacer otras a las que estamos habituados desde la zona de confort; y estas reclaman reconocimiento y transferencia. Por eso a la creatividad hay que añadirle también autoridad para introducir cambios, y tradición y transmisión para extenderlos.

\section{EL ROL DE PROFESIONALES Y ORGANIZACIONES}

Cada agente en el terreno bioético tiene que hablar desde su lugar, desde su experiencia. No es la misma la perspectiva de la filosofía, la medicina, el derecho, la piscología, la biología, el trabajo social, la enfermería, la fisioterapia, la terapia ocupacional, la odontología, etc. Uno tiene que explicitar la tradición de la que viene y la peculiaridad de la mirada que esa tradición y ese profesional aportan. No hay que dejar de ser el que se es, ni querer ser lo que no se es. La riqueza del diálogo bioético consiste en que sea plural en ideologías y perspectivas. Ahí radica su grandeza y potencia, pero también su gran dificultad.

Es difícil dialogar en contextos muy interdisciplinares y acordar una recomendación. Es importante en el diálogo multi e interdisciplinar que es la bioética que cada uno se haga fuerte en su especialidad y sepa aportar aquello que desde su disciplina le hace idóneo para participar en el comité. A lo mejor, el filósofo o el jurista consideran muy adecuada determinada práctica, y quienes tienen que llevarla a cabo, incluso estando totalmente convencidos de las razones esgrimidas por sus reflexivos 


\begin{tabular}{|c|c|}
\hline $\begin{array}{r}\text { FOLIA HUMANÍSTICA, Revista de Salud, cienc } \\
\text { Núm.2(Vol. 2) jun }\end{array}$ & $\begin{array}{l}\text { ciales y humanidades } \\
\text { 2020. ISSN 2462-2753 }\end{array}$ \\
\hline \multicolumn{2}{|l|}{ (a) } \\
\hline http://doi.org/10.30860/0064 & \\
\hline
\end{tabular}

colegas, no se sienten respaldados en sus puestos de responsabilidad para dejar de hacer, para reorganizar la tarea y poder hacer lo que estos proponen. Y precisamente lo crucial en bioética, y en ética aplicada en general, es el impacto. El riesgo es que la recomendación que hacemos los comités es muy buena; sin embargo, no puede llevarse a la práctica porque desconsideramos cambiar otros factores. $Y$ entre esos otros factores está el coraje de los médicos, enfermeras, fisioterapeutas, trabajadoras sociales, etc., para decir que, como está hoy la cosa, no se puede. A veces cabe decir un colectivo no para luego proponer cómo en esas circunstancias se podría hacer. No podemos desconsiderar el entorno laboral y organizativo donde las recomendaciones han de llevarse a cabo.

Estas deficiencias han generado cierta decepción entre sus miembros. Los profesionales que ya recibieron formación bioética se encuentran con una gran discrepancia entre lo jurídico, lo factible, personal y organizativamente, y los discursos éticos que escribimos desde los comités de ética asistenciales o nacionales y en las publicaciones bioéticas. Esto puede generar en los profesionales una visión de la bioética como algo accesorio e inútil, propio de quijotes, cuando no de gente reflexiva pero poco atenta a la realidad a transformar. Por ello es importante medir el seguimiento de las recomendaciones. Aunque lo nuestro sea la duda, la atención a las alternativas, la deliberación y la propuesta de recomendaciones no vinculantes, si nadie las sigue, o tienen muy poca incidencia, debemos repensar el porqué.

A los profesionales que sí habían sido bien formados y estaban motivados, les faltó el reconocimiento al gran trabajo en equipo que hicieron. Mas esos profesionales trabajaban con frecuencia desde un alto nivel de exigencia, casi desde el voluntariado y la motivación, y ya no dan para tanto. Los humanos no creamos en tierra hostil, nos limitamos a defendernos para sobrevivir; allí no tiene lugar la vida buena ni la posibilidad de su búsqueda.

Los Comités de ética asistenciales y los de investigación abordaron los casos, pero no lograron el cambio cultural y político. Ahí es donde más debemos incidir. Casi 


FOLIA HUMANÍSTICA, Revista de Salud, ciencias sociales y humanidades
Núm.2 (Vol. 2) junio-julio 2020. ISSN 2462-2753

nada en la vida es mecánico, precisa de animación. Contar con el voluntarismo puede llegar a ser contraproducente pasado un tiempo. Generar cultura pasa por quererla generar y por desbrozar el terreno para cultivar. Planificar y priorizar en las agendas pasa por participar. Pero para participar hace falta creer que sirva de algo. Y a los profesionales convencidos no les ayuda la sensación de ineficiencia.

Otra de las dificultades radica en abandonar la centralidad del diagnóstico y tratar a la persona como una historia de vida; abandonar pues la centralidad de los profesionales y organizaciones a los que adaptarse y focalizarse en las problemáticas de salud que, en la vida cotidiana del paciente, se manifiestan y viven subjetivamente. A eso alude el modelo centrado en la persona (ACP). Sin cultura bioética, aunque proliferen buenas iniciativas (como la ACP; el modelo de decisiones compartidas; la integración de lo sociosanitario, etc.) quedarán como anécdotas al vaivén de modas más o menos ideológicas pues el terreno es endeble, su cultivo no fructificará. Faltarán los fundamentos que toda cultura requiere, nos quedaremos en la superficie.

Nos hace falta asimismo investigación bioética verdaderamente interdisciplinar, y coordinación entre los que nos dedicamos como docentes e investigadores. Hace falta presupuesto, porque el voluntarismo de unos y el carisma de sus líderes fundan, pero no son suficientes para consolidar y hacer el mantenimiento o la extensión de la cultura.

La bioética es reflexión crítica, es diálogo deliberativo, y requiere de conocimientos, actitudes y habilidades. Los conocimientos sobre el caso los hemos intentado trasmitir a los otros profesionales involucrados. Quizás fallamos en las habilidades y destrezas sociales, comunicativas, claves en la eficiencia de la tarea de la bioética. Pero donde más énfasis deberíamos poner es en la actitud, en las maneras de ser y estar de los profesionales y las organizaciones. Seguramente porque en los niveles de grado la bioética se centró en la trasmisión de conocimientos y habilidades, algo sin duda crucial, pero no se insistió suficientemente en las actitudes. Estas nos remiten a la necesidad del entorno comunitario y a la ejemplaridad. $Y$ si en algunos 
másteres de bioética lográbamos el desarrollo de esa actitud con la formación del carácter de un tipo de profesional, a veces, como ya hemos dicho, les generamos estrés, por mayor conciencia del problema y una mayor frustración ante la soledad y falta de reconocimiento institucional.

Cuando nos dirigimos a profesionales (en ejercicio o en camino de ejercer), hay que hacerles vivir el compromiso con su profesión, desde la ineludible confianza que tendrán que generar en el paciente, que no depende ya tanto del título que se ostente, como del tipo de persona que sea el profesional, todo lo cual se aprende. Para ello, más que códigos de valores y planes estratégicos, hace falta comunidad, conciencia de pertenencia y una tradición que solo se puede transmitir por amor a la cultura.

Como la formación del carácter de los profesionales asistenciales es una formación en virtudes (Pellegrino y Thomasma, 1993), y esta depende de la cultura comunitaria, es crucial cómo esta les acoge y acompaña. El acompañamiento que reciba el estudiante en la facultad, el residente en el hospital, su acogida por el equipo en el servicio y en el centro u hospital, etc. son trascendentales. De ello dependen muchos factores: la razón de ser de las facultades (formar buenos profesionales asistenciales); la motivación y autoconfianza del profesional asistencial (se sabe preparado y con ganas); la confianza de los pacientes en esos profesionales asistenciales (eficiencia de su labor), la salud de la sociedad.

El profesional asistencial no va a poder dar lo que no es, y qué sea depende también del acompañamiento que haya recibido, de los referentes que haya tenido, del trato y acogida que haya recibido. El peligro de olvidar los fines, las razones de ser de la profesión, radica en crear cuerpos de técnicos altamente especializados $\sin$ rumbo: un cuerpo de profesionales expertos en técnicas, pero sin alma ni ánimo; la mecánica y la burocracia no cuidan. Los humanos somos de echar raíces, para lo cual hay que abonar el terreno, con los pies en el suelo (humus) y con humildad, cultivar para que otros recojan los frutos. Pero esta vez sabiendo que vamos a ejercer la 


FOLIA HUMANÍSTICA, Revista de Salud, ciencias sociales y humanidades
Núm.2 (Vol. 2) junio-julio 2020. ISSN 2462-2753

profesión con otros para otros. Es infructuoso formar a los futuros profesionales en entornos de alta competitividad individualista cuando luego tendrán que trabajar con humildad y en equipos interdisciplinares.

Las organizaciones sanitarias siguen siendo en exceso piramidales, burocráticas y rígidas. La bioética no vive bien en coordenadas egocéntricas; lo suyo es ser para otros. La autoridad moral, esa capacidad de reconocer buenas directrices y hacerlas seguir, surgen del reconocimiento, no de la imposición. Por eso no ayudó convertir causas bioéticas en puntos de la dirección por objetivos. Es crucial, más que hacer y lograr que se sepa lo que se hace, que se quiera hacer y adaptarlo al caso particular en su entorno.

La función de profesional asistencial es imposible sin vínculos, sin círculos de confianza. Por eso tan importantes como las supervisiones y las sesiones clínicas, son las deliberaciones y las reflexiones éticas. Es importante que profesional asistencial disponga de espacios donde poder reflexionar y ponderar la mejor decisión en casos de complejidad en el trato con los pacientes, los compañeros, los directivos, etc. Esta acogida afianza la conciencia de pertenencia a una comunidad y retroalimenta la motivación que redunda en la calidad asistencial.

El individualismo liberal, la arbitrariedad en la cartera de servicios según autonomías, administraciones y sus correspondientes vaivenes ideológicos forman parte de estas dimensiones culturales a las que la bioética debe, finalmente, prestar más atención. La viabilidad del sistema de salud pasa por contar con una cultura bioética como factor clave de su legitimidad y credibilidad. Pero esta vez no basta con tener comités o asignaturas en grados y postgrados, habrá que predicar con el ejemplo. Ahora bien, la ejemplaridad no alude solo a la persona, sino a la del equipo e institución en la que se está. La transversalidad exige y genera equipo, clave para fomentar cultura: conocimientos, prácticas, habilidades, actitudes.

La comunidad y la cultura que siembran estas condiciones sociológicas y psicológicas son trascendentales para la formación de los profesionales asistenciales. 


\begin{tabular}{l}
$\begin{array}{l}\text { FOLIA HUMANISTICA, Revista de Salud, ciencias sociales y humanidades } \\
\text { Núm.2 (Vol. 2) junio-julio 2020. ISSN 2462-2753 }\end{array}$ \\
$\begin{array}{l}\text { Mas allá de los Comités de Ética Asistencial: hacia una cultura bioética } \\
\text { http://doi.org/10.30860/0064 }\end{array}$ \\
\hline
\end{tabular}

Esta es responsabilidad de las organizaciones que los contratan, para que no dañen a quienes reciben, para que no los erosionen, durante la práctica profesional, perdiendo lo esencial y nuclear de la profesión y la misión de las ciencias de la salud.

La trasversalidad y la trascendentalidad que caracteriza a la bioética reclaman coordinación y comisiones porque tienen una finalidad común y hay que delegar responsabilidades. De la misma manera que no se ha sabido ligar bien lo bio-psicosocial que el concepto de salud engloba, tampoco se han coordinado los profesionales, servicios e instituciones que la ciudadanía necesita. Necesitamos liderazgos en reflexión crítica de profesionales involucrados, respaldados y reconocidos por sus respectivas instituciones. Pero eso pasa por tener directivos que se toman en serio los valores y los transforman en políticas éticas, muchas de las cuales derivan de las recomendaciones de los comités de ética a los que deberían escuchar y preguntar más.

Por eso debemos prestar atención a la eficiencia, a que las recomendaciones cuajen en políticas con directrices sobre cómo y quiénes llevarlas a acabo. De otro modo, caemos en las éticas de la convicción, traicionando la responsabilidad acerca del encargo que se nos hizo. Por supuesto que lo propio de la bioética es el nivel de la reflexión y que el activismo es otra cosa. Pero que la reflexión no se atenga a las dificultades y a los obstáculos a remover, quita a la bioética la condición de ética que se aplica. Asimismo, desconsiderar el contexto en el que llevar a cabo las recomendaciones, nos condena a la anécdota que no deviene categoría, ni hábito ni cultura.

\section{LA CIUDADANÍA Y LOS POLÍTICOS}

Hemos visto cómo en las facultades de las ciencias de la salud hemos introducido asignaturas de bioética, deontología y derecho, mas no basta con la buena voluntad, ni siquiera con la vocación de médico o enfermera. Hace falta una cultura que vele por acompañar al profesional asistencial es su proceso de llegar a serlo y en el proceso de que su ejercicio no lo corrompa. Eso depende a su vez del anclaje en 


FOLIA HUMANISTICA, Revista de Salud, ciencias sociales y humanidades
Núm.2 (Vol. 2) junio-julio 2020. ISSN 2462-2753

la tradición y en la cultura de la organización. No es mero sentido común; este no deja de ser un sentido cultural que se forja en una comunidad que trasmite capacidad reflexiva y deliberativa. Sin embargo, la actual cultura sanitaria, tan gestionada, tan exigente, tan demandadora, puede que ponga en peligro esos supuestos, por eso era importante hablar de ellos y explicitarlos: que ejercemos para la ciudadanía y dependemos de su confianza.

Ahora bien, con cultura bioética no sólo nos referimos a una cultura organizativa de muros hacia dentro, a nivel meso, también aludimos a la relación entre los departamentos (de salud, de servicios sociales, de educación, etc.), de estos con sus comités, o con los parlamentos. Asimismo, cabe involucrar a la ciudadanía, para que participe en la agenda de los problemas bioéticos que se quiere abordar. $Y$ también los políticos y gestores tienen que asumir pragmáticamente (centrados en problemas reales de los pacientes) la necesidad inmensa de cambios que no pueden hacer sin la participación de profesionales y pacientes, democráticamente, es decir, dialógicamente. Pero si a nivel más interno cuesta, más cuesta hacer llegar los acuerdos y razones a los políticos y a la ciudadanía.

A nivel del impacto que la bioética ha tenido en los políticos y los gestores, el resultado es negativo. Los parlamentos van a tener que legislar sobre eutanasia, sobre consumo de cannabis, sobre nuevas técnicas de reproducción asistida, etc., donde va a ser necesario el diálogo. Si ponemos y disponemos de comités de ética, deberían consultarnos y escucharlos más a la hora de proponer políticas desde la ética cívica, y no desde las ideologías partidistas. Muchas reflexiones bioéticas se dirigían a ellos, a que convirtieran en políticas nuestras recomendaciones éticas; y no hicimos del todo bien esa cadena de transmisión de conocimientos bioéticos para que propiciaran cambios legislativos y organizativos en la atención de la salud de la ciudadanía.

Si bien mejoramos la presencia de temas bioéticos en los medios de comunicación, desarrollando las pertinentes habilidades, no logramos el gran impacto en la ciudadanía. La atención centrada en lo biológico y lo patológico ganó peso 


FOLIA HUMANÍSTICA, Revista de Salud, ciencias sociales y humanidades
Núm.2 (Vol. 2) junio-julio 2020. ISSN 2462-2753

respecto a las historias de vida y a la visión integral biopsicosocial que Engel reclamaba (Engel, 1977). Nos gusta pensar que algo de incidencia de la bioética en los servicios debe notar la ciudadanía, pero intuimos que sobre todo lo constatan en la firma de papeles que les suena más a profesiones a la defensiva (a la defensiva de los pacientes, de las quejas, de los requerimientos judiciales) que a verdadera participación en el proceso en el que están. No hemos trasmitido el conocimiento a la ciudadanía con ánimo de empoderarla para tomar decisiones sobre el modelo de salud, más allá, aunque también, de su caso particular.

La ciudadanía es la destinataria última de todo este quehacer bioético. No aludimos sólo pues a la dificultad de incorporar el miembro lego en los CEA's (Ramos, Morlans, 2005); sino a incorporar a la ciudadanía, y a los profesionales, en las tomas de decisiones sobre el sistema sanitario y sobre las agendas de políticas de salud. Hay que propiciar y buscar la participación de la ciudadanía, pero hay que motivarla. Y para motivar lo adecuado es constatar la necesidad. En salud lo tenemos fácil, nos suele importar a todos; lo que va a costar es hacerles confiar en que su participación será tenida en cuenta. Esto incumbe también al trabajo de profesionales elaborando estrategias, planes, informes varios sobre cómo mejorar el servicio.

Para hacer llegar nuestras reflexiones a los ciudadanos, y que confíen en el juicio ponderado que en los comités queremos dar, necesitamos cambiar el género narrativo. Nos ha faltado creatividad; el cine y la literatura lo han hecho mejor que nuestros sesudos documentos que, sin querer ser tratados académicos, casi acaban siendo leídos por el mismo gremio bioético que los hemos elaborado. Trabajar con los gabinetes de comunicación y encontrar el canal y los nuevos formatos en la era mediática (no sólo las conferencias y los escritos) es otro de los puntos que la cultura bioética tiene que mejorar si quiere llegar a la ciudadanía y lograr su participación.

Una cultura bioética exige distribución de recursos limitados, sí, pero también de reconocimiento (Honneth, 1997). Es de justicia dar reconocimiento. Esto significa atender desde un paradigma de pluralismo y hacer participar a profesionales y 


FOLIA HUMANÍSTICA, Revista de Salud, ciencias sociales y humanidades
Núm.2 (Vol. 2) junio-julio 2020. ISSN 2462-2753

ciudadanos, más allá de la salud privada, en los asuntos públicos. La cultura sanitaria sigue siendo en exceso vertical y jerárquica, el cambio de paradigma de autonomía del paciente, de la centralidad de este como ciudadano, es mucho más exigente que la mera participación del enfermo y sus allegados en su propio proceso. Hay que esquivar igualmente incurrir en una mera relación clientelar de quien paga manda.

La bioética es constitutivamente democrática, en tanto que espacio de encuentro dialógico y deliberativo entre los afectados por las decisiones; y democratizarla pasa por discutir públicamente procesos y temáticas. Se trata de ser críticos desde la postconvencionalidad que cuestiona determinadas convenciones, para removerlas y para cambiarlas creando otras maneras de hacer, dadas las nuevas posibilidades técnicas o las trasformaciones de las dinámicas sociales en sociedades pluralistas y aceleradas. Ese es el quehacer de la bioética y ese es su contexto, encontrar la coexistencia entre el uso público y privado de la razón, por los cuales se mantiene una organización en su quehacer cotidiano, al mismo tiempo que se la hace avanzar al estar atentos a sus deficiencias y necesidad de mejora (Kant, 2000).

La salud es más que lo sanitario y el caso personal, es también social, comunitaria, y cultural. Siguiendo la razón vital (la bioética comparte etimología con ella) de Ortega y Gasset, cambiar las circunstancias es cambiarnos a nosotros (Ortega, 2014). En este cambio cultural lo social y lo sanitario no pueden continuar en unos esquemas que han dañado en exceso lo comunitario, la consciencia de pertenencia a algo común, y la confianza en lo público. Vale la pena recuperar una visión más integral, más holística de la bioética, como ética de la vida en toda su magnitud y amplitud. Y no sólo porque la salud no se reduce a lo sanitario; o porque los factores sociales determinantes de aquella son temas bioéticos, sino porque en la aldea global debemos trabajar y pensar juntos ${ }^{7}$.

\footnotetext{
7 Tenemos esperanza en la recién creada Red de Comités de Ética de la Generalitat de Cataluña: a ver si somos capaces de transferirnos conocimientos y mejorar nuestra eficiencia la creación de políticas para y con la ciudadanía, de la que somos parte.
} 
En absoluto queremos desconsiderar los importantes cambios culturales que la bioética española ha generado: desde la creación de comités que funcionan, al implacable impacto en la cultura paternalista previa reinante durante siglos ( $y$ todo ello en pocos años). No cabe duda que los profesionales de la salud han interiorizado que la ciudadanía tiene derechos (a ser informada, a participar en la toma de decisiones, a rechazar tratamientos etc.) y que, aun siendo exigencia jurídica con procedimientos un tanto burocráticos, los profesionales no pueden intervenir en su salud sin su permiso.

No obstante, hemos de ir más allá, más allá de lo clínico asistencial, a lo global centrado en la vida (bio-ética), una vida que es narrativa, existencial, contextualizada e interdependiente. Hay que tomar conciencia del cuidado del mundo, de mantenerlo y regenerarlo. Por eso, más allá de profesionales convencidos y bien formados, necesitamos cultura, políticas, estructuras básicas institucionales flexibles ágiles, pensadas y pensantes, muy alejadas de lo burocrático y medieval. Tenemos razones para creer que podemos, y como la realidad, además, lo exige, seguiremos pensando para resolver problemas. Esta vez el problema es de cambio cultural de gran calado para estar a la altura de las nuevas circunstancias.

\section{BIBLIOGRAFÍA}

Arendt, H (2016). La condición humana. Barcelona: Paidós

Engel, G. (1977). The Need for a New Medical Model: a Challenge for Biomedicine. Science. 196:129-36.

Falcó Pegueroles, Anna M (2012). Análisis de la conflictividad ética en los profesionales de enfermería en las unidades de cuidados paliativos. Tesis doctoral Universidad de Barcelona. https://www.tdx.cat/bitstream/handle/10803/84082/AMFP TESIS.pdf.

Honneth, A (1997). La lucha por el reconocimiento. Barcelona: Crítica. 
FOLIA HUMANÍSTICA, Revista de Salud, ciencias sociales y humanidades

Núm.2 (Vol. 2) junio-julio 2020. ISSN 2462-2753

Mas allá de los Comités de Ética Asistencial: hacia una cultura bioética

Begoña Román Maestre

http://doi.org/10.30860/0064

Kant, I (2000). ¿Qué es Ilustración? en Filosofía de la Historia. Madrid: Fondo de Cultura

Económica.

Maclntyre, A (1987). Tras la virtud. Barcelona: Crítica.

Ortega y Gasset, J (2014). Meditaciones del Quijote y otros ensayos. Madrid: Alianza.

Pellegrino, E \& Thomasma, D (1993). The Virtues in Medical Practice, Oxford University Press,

Ramos, S y Morlans, M (2011). Legos en el comité de ética: una reflexión desde la ética dialógica. Perspectivas bioéticas, Revista de Bioética y Derecho. Universitat de Barcelona. http://revistes.ub.edu/index.php/RBD/article/view/7740

Red de Comités de ética de Cataluña:

http://politiquesdigitals.gencat.cat/ca/pgov_ambits_d_actuacio/administracio-

publica/eticapublica/xarxacomites/

Wilson, E.O (2011):

https://www.lavanguardia.com/magazine/20110729/54192495692/edward-wilson-el-serhumano-del-futuro-sera-sorprendente.html

\section{Begoña Román}

Profesora de Filosofía de la Universidad de Barcelona

Presidenta del Comité de Ética de Servicios Sociales de Catalunya.

Vocal del Comité de Bioética de Catalunya.

broman@ub.edu

\section{Cómo citar este artículo:}

Román, B., "Más allá de los Comités de Ética Asistencial: hacia una cultura bioética", Folia Humanística, 2020; 2(2):1-17. Doi: http://dox.doi.org/10.30860/0064

(c) 2020 Todos los derechos reservados a la Revista Folia Humanística de la Fundación Letamendi Forns. This is an open access article. 